\title{
The Relativistic Problem of Motion in Co-moving Co-ordinates *
}

\author{
ENRICO MASSA \\ Istituto Matematico dell'Università di Genova
}

Received May 15, 1971

\begin{abstract}
The role of co-moving atlases is discussed in connection with a possible formulation of the problem of motion in General Relativity. The concept of co-moving scheme is defined and applied to various cases of physical interest. In particular in the Einstein-Maxwell case, we derive a general uniqueness proof for the Maxwell equations.

The dynamical meaning of the equation $T^{i j}{ }_{1 / j}=0$ is proved, and a scheme for the solution of the problem of motion in co-moving co-ordinates is proposed.
\end{abstract}

\section{§ 1. Introduction}

In a previous paper [1] we have shown how the problem of motion for a material continuum in General Relativity may be conveniently formulated in terms of the projection operators associated to the congruence $\Gamma$ of stream-lines of the continuum itself.

Our results were obtained under the hypothesis $\Gamma \in\left(C_{1}, \tilde{C}_{2}\right)^{1}$, a condition which is general enough to include every physical situation, with the only exception of shock waves [2-4].

However, almost all problems of actual physical interest may be treated under the stronger condition $\Gamma \in\left(C_{2}, \tilde{C}_{4}\right)$.

In this case, it is always possible to choose local co-ordinates $\left(x^{1}, x^{2}, x^{3}, x^{4}\right)$ in the space-time manifold $\mathscr{V}_{4}$ in such a way that the curves of $\Gamma$ have local equation $x^{4}=$ var.

We call such a collection of local co-ordinates a co-moving atlas.

The use of co-moving atlases simplifies the structure of the problem of motion, and makes the whole formalism introduced in [1] more transparent.

The object of the present paper is to discuss this particular view point, and to indicate its implications.

* Lavoro eseguito nell'ambito dell'attività dei Gruppi di Ricerca Matematica del Consiglio Nazionale delle Ricerche.

${ }_{1}$ The notation $\tilde{C}_{n}$ stands for "piecewise $C_{n}$ "; the notation $\left(C_{m}, \tilde{C}_{n}\right)(m<n)$ for " $C_{m}$, piecewise $C_{n}{ }^{\prime \prime}$. 
In Subsection 2.1 we show that there is a natural correspondence between the determination of a $\left(C_{2}, \tilde{C}_{4}\right)$ congruence of time-like curves, and the selection of a suitable class of admissible atlases in $\mathscr{V}_{4}$.

We also show that this selection may always be accomplished by means of a set of co-ordinate conditions invariant under the group $\mathscr{I}$ of internal transformations ${ }^{2}[5,6]$

$$
\left\{\begin{array}{l}
\bar{x}^{\alpha}=\bar{x}^{\alpha}\left(x^{1}, x^{2}, x^{3}\right), \\
\bar{x}^{4}=\bar{x}^{4}\left(x^{1}, x^{2}, x^{3}, x^{4}\right) .
\end{array}\right.
$$

In Subsection 2.2 we introduce the concept of space tensor [7-9] and analyse its properties.

These results are used in Section 3 to formulate the problem of motion in terms of co-moving atlases. We introduce the so-called co-moving scheme (Subsection 3.1) and apply it to an arbitrary material continuum (Subsection 3.2) and to an electrically charged dust (Subsection 3.3). In the latter case, we also discuss the mathematical structure of Maxwell's equations in the co-moving scheme.

Finally, in Section 4, we derive the equations of motion for both types of continua discussed in Section 3, and propose a practical scheme for the effective solution of the problem of motion.

\section{§ 2. Mathematical Preliminaries}

\subsection{The Physical Frame of Reference Associated to a Given Congruence of Curves}

Let $\mathscr{A}=\left\{\left(U_{(a)}, h_{(a)}\right), a \in N\right\}$ be a physically admissible atlas in the manifold $\mathscr{V}_{4}{ }^{3}[10]$. By choosing a suitable refinement of $\mathscr{A}$ (if necessary), we may always assume that all the sets $h_{(a)}\left(U_{(a)}\right)$ are open connected subsets of the euclidean four-space $E^{4}[11]$.

We denote by $\left(x_{(a)}^{1}, x_{(a)}^{2}, x_{(a)}^{3}, x_{(a)}^{4}\right)$ the local co-ordinates defined by the $\operatorname{map} h_{(a)}$. In each co-ordinate neighbourhood $U_{(a)}$, let $\Gamma_{(a)}$ denote the congruence of co-ordinate lines $x_{(a)}^{4}=$ var. All congruences $\Gamma_{(a)}$ are clearly $\left(C_{2}, \tilde{C}_{4}\right)$ and time-like. Each congruence $\Gamma_{(a)}$ defines in $U_{(a)}$ a unique field $\gamma$ of unit tangents, whose expression in local co-ordinates is (a)

$$
\underset{(a)}{\gamma^{i}}=\frac{\delta_{4}^{i}}{\sqrt{-g_{44}}} ; \quad \underset{(a)}{\gamma_{i}}=\frac{g_{i 4}}{\sqrt{-g_{44}}} .
$$

${ }^{2}$ Unless otherwise stated, Latin indices will run from 1 to 4 , and Greek indices from 1 to 3. Obviously, this convention will not apply to the subscripts $(a)$, (b), etc. used in Section 2 to label the various charts of the atlas $\mathscr{A}$, as these subscripts are not tensor indices.

3 We recall that an atlas $\mathscr{A}$ in $\mathscr{V}_{4}$ is physically admissible if and only if in every chart $\left(U_{(a)}, h_{(a)}\right) \in \mathscr{A}$ the coefficients $g_{i j}$ of the metric are $\left(C_{1}, \tilde{C}_{3}\right)$ and satisfy the inequalities $g_{44}<0,\left|g^{\alpha \beta}\right|$ positive definite. 
Now, let $\Omega$ be a connected domain of $\mathscr{V}_{4}$. Then, a necessary and sufficient condition that the fields $\{\underset{(a)}{\gamma}, a \in N\}$ may be pieced together to form a well defined vector field $\gamma$ over the whole of $\Omega$ is that, for every $a, b \in N$, the equation

$$
\gamma_{(a)}^{i}=\gamma_{(b)}^{j} \frac{\partial x_{(a)}^{i}}{\partial x_{(b)}^{j}}
$$

be satisfied in the overlap $U_{(a)} \cap U_{(b)} \cap \Omega^{4}$.

Notice that, whenever this condition is satisfied, the congruences $\Gamma_{(a)}$ themselves may be pieced together to form a unique $\left(C_{2}, \tilde{C}_{4}\right)$ time-like congruence $\Gamma$ defined over the whole of $\Omega$. In this case we say that the atlas $\mathscr{A}$ is adapted to $\Gamma$. Notice also that, if Eq. (2.2) is satisfied for a given choice of $\Omega$, then (by continuity), it is also satisfied if we replace $\Omega$ by $\bar{\Omega}$. Therefore, we may always assume that the domain $\Omega$ is closed.

Using Eq. (2.1), we see that Eq. (2.2) is mathematically equivalent to the condition that for every $a, b \in N$, the transformation of local co-ordinates $h_{(a)} h_{(b)}^{-1}$ satisfy $\frac{\partial x_{(a)}^{\alpha}}{\partial x_{(b)}^{4}}=0$ at every point $x \in h_{(b)}\left(U_{(a)} \cap U_{(b)} \cap \Omega\right)$. Transformations of the form

$$
\left\{\begin{array}{l}
\bar{x}^{\alpha}=\bar{x}^{\alpha}\left(x^{1}, x^{2}, x^{3}\right), \\
\bar{x}^{4}=\bar{x}^{4}\left(x^{1}, x^{2}, x^{3}, x^{4}\right)
\end{array}\right.
$$

constitute a group $\mathscr{I}$, called the group of internal transformations. We can therefore state

Theorem 2.1. A physically admissible atlas $\mathscr{A}$ defines a unique $\left(C_{2}, \tilde{C}_{4}\right)$ time-like congruence $\Gamma$ in a closed connected domain $\Omega \subset \mathscr{V}_{4}$ if and only if for every pair of charts $\left(U_{(a)}, h_{(a)}\right),\left(U_{(b)}, h_{(b)}\right) \in \mathscr{A}$, the transformation

$$
h_{(a)} h_{(b)}^{-1}: h_{(b)}\left(U_{(a)} \cap U_{(b)} \cap \Omega\right) \rightarrow E^{4}
$$

is an internal transformation.

We now try to invert the result stated in Theorem 3.1.

Let $\Gamma$ be a $\left(C_{2}, \tilde{C}_{4}\right)$ time-like congruence defined in a domain $\Omega \subset \mathscr{V}_{4}$.

We denote by $[\Gamma]$ the totality of physically admissible atlases adapted to $\Gamma$, and call it the physical frame of reference associated to the congruence $\Gamma$. Then, once the domain $\Omega$ has been fixed, the knowledge of the congruence $\Gamma$ is mathematically equivalent to the knowledge of the class $[\Gamma]$.

To identify the latter, we need the concept of co-ordinate conditions.

A set $\mathscr{C}$ of co-ordinate conditions is a collection of $m(m \leqq 4)$ equations of non tensorial character, which do not impose any restriction on the geometry of $\mathscr{V}_{4}$, but only on the choice of the local co-ordinates.

\footnotetext{
${ }^{4}$ Obviously, we regard Eq. (2.2) as an identity whenever $U_{(a)} \cap U_{(b)} \cap \Omega=\emptyset$.
} 
For a given set $\mathscr{C}$ of co-ordinate conditions, we denote by [ $\mathscr{C}]$ the totality of physically admissible atlases consistent with $\mathscr{C}$. Then, $\mathscr{C}$ itself is physically admissible if and only if $[\mathscr{C}]$ is not empty. In the latter case, we introduce the set $G(\mathscr{C})$ of all the transformations between the local co-ordinates associated to the atlases $\mathscr{A} \in[\mathscr{C}]$.

Theorem 2.2. Every $\left(C_{2}, \tilde{C}_{4}\right)$ time-like congruence defined in a domain $\Omega \subset \mathscr{V}_{4}$ identifies in $\Omega$ a unique set $\mathscr{C}$ of admissible co-ordinate conditions such that $G(\mathscr{C})=\mathscr{I}$ and $[\mathscr{C}]=[\Gamma]$. Conversely, given any admissible set $\mathscr{C}$ of co-ordinate conditions in $\Omega$, satisfying $G(\mathscr{C})=\mathscr{I}$, there is a unique congruence $\Gamma$ defined in $\Omega$ and such that $[\Gamma]=[\mathscr{C}]$.

Proof. Let $\Gamma$ be a $\left(C_{2}, \tilde{C}_{4}\right)$ time-like congruence in $\Omega$. Consider the field $\lambda$ of unit tangents to the lines of $\Gamma$. Then, by Eq. (2.1), an atlas $\mathscr{A}$ is adapted to $\Gamma$ if and only if in every chart of $\mathscr{A}$ the condition

$$
\lambda^{\alpha}=0
$$

is satisfied at every point of $\Omega$. Our assumptions ensure that Eqs. (2.5) constitute a set $\mathscr{C}$ of physically admissible co-ordinate conditions in $\Omega$. We have thus $[\mathscr{C}]=[\Gamma]$. The condition $G(\mathscr{C})=\mathscr{I}$ is also verified by direct computation. This proves the first part of Theorem 2.2.

Conversely, let $\mathscr{C}$ be an admissible set of co-ordinate conditions in $\Omega$, satisfying $G(\mathscr{C})=\mathscr{I}$. Then, for every atlas $\mathscr{A} \in[\mathscr{C}]$ and every pair of charts $\left(U_{(a)}, h_{(a)}\right),\left(U_{(b)}, h_{(b)}\right) \in \mathscr{A}$, the transformation $h_{(a)} h_{(b)}^{-1}: h_{(b)}\left(U_{(a)} \cap U_{(b)}\right.$ $\cap \Omega) \rightarrow E^{4}$ belongs to $G(\mathscr{C})$ (by definition), and is therefore an internal transformation. By Theorem 2.1, this implies that the atlas $\mathscr{A}$ defines a unique time-like congruence $\Gamma_{\mathscr{A}}$ in $\Omega$.

Moreover, let $\mathscr{A}_{1}, \mathscr{A}_{2} \in[\mathscr{C}]$. Then, it may be easily checked that also $\mathscr{B}=\mathscr{A}_{1} \cup \mathscr{A}_{2} \in[\mathscr{C}]^{5}$.

Therefore, by the previous argument, $\mathscr{B}$ defines a unique congruence $\Gamma_{\mathscr{B}}$ in $\Omega$. In view of the definition of $\mathscr{B}$ (see footnote 5) this implies $\Gamma_{\mathscr{A}_{1}}=\Gamma_{\mathscr{A}_{2}}=\Gamma_{\mathscr{B}}$ def $=\Gamma$. We have thus $[\mathscr{C}]=[\Gamma]$, and the proof of Theorem 2.2 is complete.

An immediate consequence of Theorem 2.2 is the following

Corollary 2.1. The determination of a $\left(C_{2}, \tilde{C}_{4}\right)$ time like congruence $\Gamma$ in $\Omega$ is mathematically equivalent to the determination of a set $\mathscr{C}$ of co-ordinate conditions defined in $\Omega$ and invariant under the group of internal transformations.

${ }^{5}$ We recall that, if $\mathscr{A}_{1}=\left\{\left(U_{(a)}, h_{(a)}\right)\right\}$ and $\mathscr{A}_{2}=\left\{\left(V_{(b)}, k_{(b)}\right)\right\}$, the union $\mathscr{A}_{1} \cup \mathscr{A}_{2}$ is the atlas $\mathscr{B}=\left\{\left(W_{(c)}, z_{(c)}\right)\right\}$ defined by $\left(W_{(c)}, z_{(c)}\right)=\left(U_{(n)}, h_{(n)}\right)$ for $c=2 n-1$ and $\left(W_{(c)}, z_{(c)}\right)$ $=\left(V_{(n)}, k_{(n)}\right)$ for $c=2 n[11]$. 


\subsection{Space Tensors}

Let $[\Gamma]$ be the physical frame of reference associated to a $\left(C_{2}, \tilde{C}_{4}\right)$ time-like congruence $\Gamma$. The group $\mathscr{I}$ acts transitively on $[\Gamma]$ : for every pair of atlases $\mathscr{A}_{1}, \mathscr{A}_{2} \in[\Gamma]$, the transformation of local co-ordinates between $\mathscr{A}_{1}$ and $\mathscr{A}_{2}$ belongs to $\mathscr{I}$ and conversely.

Definition 2.1. A space tensor $\tilde{T}$ is a quantity that behaves like a three-tensor under the transformations (2.3), and like a scalar under the transformations (2.4).

It may be easily proved that every four-tensor may be resolved into a suitable collection of space tensors by means of a natural projection technique.

Therefore, space tensors may be used instead of four-tensors in the formulation of physical laws in the frame of reference $[\Gamma]$.

The projection technique indicated above has been thoroughly investigated by Cattaneo [6-8] (see also Ref. [9]). Here we only quote some elementary results that are relevant to the subsequent discussion, and refer the reader to the Bibliography for further information.

(i) Given any contravariant four-tensor $T^{i_{1} \ldots i_{r}}$, any quantity obtained by saturating $p$ indices of $T^{i_{1} \ldots i_{r}}(0 \leqq p \leqq r)$ with the vector field $\gamma_{i}$ defined by Eq. (2.1), and letting the remaining $r-p$ indices run from 1 to 3 is a contravariant space tensor of rank $r-p$. Thus, for example, the tensor $T^{i j}$ generates four space tensors of rank 2,1 and 0 , namely $T^{\alpha \beta}$, $\gamma_{i} T^{i \alpha}=\frac{T_{4}^{\alpha}}{\sqrt{-g_{44}}}, \gamma_{j} T^{\alpha j}=\frac{T_{4}^{\alpha}}{\sqrt{-g_{44}}}$ and $\gamma_{i} \gamma_{j} T^{i j}=-\frac{T_{44}}{g_{44}}$.

(ii) The previous procedure, applied to the metric tensor $g^{i j}$, yelds only one non trivial space tensor, namely $g^{\alpha \beta}$. We take $g^{\alpha \beta}$ as the contravariant metric tensor in the reference $[\Gamma]$, and define the corresponding covariant metric tensor $\gamma_{\alpha \beta}$ by the condition $g^{\alpha \lambda} \gamma_{\lambda \beta}=\delta_{\beta}^{\alpha}$.

This yields

$$
\gamma_{\alpha \beta}=g_{\alpha \beta}+\gamma_{\alpha} \gamma_{\beta} \text {. }
$$

With the aid of $\gamma_{\alpha \beta}$ we may now lower the indices of every space tensor obtained in (i)

(iii) The spatial Ricci tensor $\tilde{\eta}_{\lambda \mu \nu}$ is defined by

$$
\tilde{\eta}_{\lambda \mu \nu}=\sqrt{\gamma} \varepsilon_{\lambda \mu \nu}
$$

where $\gamma=\operatorname{det}\left(\gamma_{\alpha \beta}\right)$ and $\varepsilon_{\lambda \mu \nu}$ is the permutation symbol. We have then

$$
\tilde{\eta}^{\lambda \mu \nu}=\frac{1}{\sqrt{\gamma}} \varepsilon_{\lambda \mu \nu}
$$


as may be easily checked. We let the reader verify that $\tilde{\eta}^{\lambda \mu v}$ is (apart from sign) the only non trivial space tensor obtained from the Ricci tensor $\eta^{i j k l}$ in $\mathscr{V}_{4}$ through the process indicated in (i).

\section{§ 3. Formulation of the Problem of Motion}

\subsection{The Co-moving Scheme}

Let $T_{i j}$ be the energy-momentum tensor of a material continuum whose hystory is contained in a world tube $\Omega \subset \mathscr{V}_{4}$.

Let the hypersurfaces $S$ - with local equation $f\left(x^{1}, x^{2}, x^{3}, x^{4}\right)=0-$ and $B$ - with local equation $x^{4}=0$ - be the boundary of $\Omega$ and a spatial section of $\Omega$ respectively. We denote by $\mu$ and $V^{i}$ respectively the density and four-velocity of the continuum, and by $\Gamma$ the corresponding congruence of stream-lines (these being world-lines having $V^{i}$ for unit tangents).

The object of the problem of motion is to determine $\mu$ and $\Gamma$ inside $\Omega$, in terms of a given set of initial and boundary data specified on the hypersurfaces $B$ and $S$. In general, this requires also the determination of the geometry of space-time inside $\Omega$.

To achieve this goal, we have at our disposal Einstein's field equations, and an auxiliary set of equations related to the physical structure of the continuum. We shall now indicate how the mathematical results obtained in Section 2 may be conveniently applied to the discussion of this problem.

We start with the tentative hypothesis $\Gamma \in\left(C_{2}, \tilde{C}_{4}\right)^{6}$. Then, according to Theorem 2.2, the class $[\Gamma]$ of admissible atlases adapted to $\Gamma$ is not empty.

We call $[\Gamma]$ the co-moving frame of reference associated to the given continuum. In view of the discussion in Section 2.1, we may regard the problem of motion as consisting of the determination of the density $\mu$ and of the co-moving frame $[\Gamma]$.

By Theorem 2.2 , this may be achieved by determining $\mu$ and a set $\mathscr{C}$ of admissible co-ordinate conditions defined in $\Omega$ and satisfying $G(\mathscr{C})=\mathscr{I}$ and $[\mathscr{C}]=[\Gamma]$. Finally, as the class $[\Gamma]$ is already completely characterized by the condition

$$
V^{\alpha}=0
$$

we see that the conditions in $\mathscr{C}$ must be mathematically equivalent to Eq. (3.1). We have now two possibilities: we may either analyse the

${ }^{6}$ Notice that we cannot take this hypothesis for granted $a$-priori, as the congruence $\Gamma$ is one of the unknowns of our problem, and has to be determined in terms of the data at our disposal. Therefore, for the time being, we may only suppose $\Gamma \in\left(C_{2}, \tilde{C}_{4}\right)$. Later on, we shall be able to discuss the legitimacy of this hypothesis. 
problem of motion in the usual way, and determine $\mu$ and $\Gamma$ in terms of the data at our disposal. In this case, the determination of the comoving frame of reference $[\Gamma]$ is rather academic, and is trivially accomplished by means of Eq. (3.1), which are now an effective set of co-ordinate conditions.

Or we may adopt what we call the co-moving scheme, which consists of formulating everything in the co-moving frame of reference $[\Gamma]$ from the very beginning. The situation in then reversed: the congruence $\Gamma$ of stream-lines of the continuum in $[\Gamma]$ is no longer an unknown, as it coincides with the congruence of co-ordinate lines $x^{4}=$ var. in any atlas $\mathscr{A} \in[\Gamma]$.

On the other hand, the co-moving frame of reference $[\Gamma]$ is no longer determined by Eq. (3.1) (which are now identically satisfied by definition), so that we have to look for a set $\mathscr{C}$ of three effective co-ordinate conditions able to identify $[\Gamma]$. If we compare this fact with the previous formulation of the problem of motion, we conclude.

Theorem 3.1. In the co-moving scheme, the problem of motion for a material continuum is reduced to the determination of the density $\mu$ and of a set $\mathscr{C}$ of effective co-ordinate conditions satisfying $G(\mathscr{C})=\mathscr{I}$ and $[\mathscr{C}]=[\Gamma]$.

Once the problem has been solved, the conditions in $\mathscr{C}$ must be mathematically equivalent to Eq. (3.1).

Definition 3.1. The equations of motion for a material continuum in the co-moving scheme are a set of four (partial differential) equations satisfying the following properties:

(i) they express the relevant kinematical quantities $\mu$ and $\mathscr{C}$ in terms of the dynamical effects described by the fields $g_{i j}$;

(ii) the remain non-trivial (i.e. they do not reduce to identities) if we neglect the local gravitational effects by letting the gravitational constant $k \rightarrow 0$ in $\Omega^{7}$.

We shall now apply the co-moving scheme to various cases of physical interest. Notice that, in accepting this scheme, we are explicitly giving up general covariance. However, we still require covariance under the group $\mathscr{I}$ of internal transformations, so that we are entitled to use the results stated in Subsection 2.2.

\subsection{The Purely Gravitational Case}

For a large class of continua, the four-velocity field $V^{i}$ and density $\mu$ are related to the energy-momentum tensor $T_{i j}$ by the eigenvalue

\footnotetext{
${ }^{7}$ The reason for this condition has already been discussed in Ref. [1].
} 
equation $[12]^{8}$

with normalization

$$
\left(T_{i j}+\mu g_{i j}\right) V^{j}=0,
$$

$$
g_{i j} V^{i} V^{j}=-1 \text {. }
$$

We assume that the eigenvalue $\mu$ exists and is real, positive and non degenerate. In this case, the energy-momentum tensor $T_{i j}$ admits a unique decomposition of the form

with

$$
T_{i j}=\mu V_{i} V_{j}-S_{i j}
$$

$$
S_{i j} V^{j}=0 .
$$

The tensor $S_{i j}$ defined by Eq. (3.5) is called the stress-tensor of the continuum. This tensor is usually a function of the velocity field $V^{i}$, of the metric tensor $g_{i j}$, and of $n$ other quantities $\lambda_{1}, \ldots, \lambda_{n}$ (pressure, viscosity, elastic constants, etc.) which depend on the physical structure of the continuum [13]. Moreover, in every physically definite situation, the quantities $\lambda_{1}, \ldots, \lambda_{n}$ are not independent, but are in fact related to one another and to $\mu, V^{i}$ and $g_{i j}$ by $n$ state equations

$$
g_{\xi}\left(\mu, V^{i}, g_{i j}, \lambda_{1}, \ldots, \lambda_{n}\right)=0 \quad(\xi=1,2, \ldots, n) .
$$

We regard the theoretical expression for $S_{i j}$ in terms of the physical parameters

$$
S_{i j}=S_{i j}\left(V^{i}, g_{i j}, \lambda_{1}, \ldots, \lambda_{n}\right)
$$

and the state equations (3.6) as data for the problem in study.

These equations may be written in the equivalent form

$$
\begin{gathered}
\lambda_{\xi}=\lambda_{\xi}\left(\mu, V^{i}, g_{i j}\right) \quad(\xi=1,2, \ldots, n), \\
S_{i j}=S_{i j}\left(\mu, V^{i}, g_{i j}\right),
\end{gathered}
$$

which is more convenient for the subsequent discussion.

In the co-moving scheme we are entitled to write

$$
V^{i}=\gamma^{i}
$$

the components $\gamma^{i}$ being defined by Eq. (2.1).

Then Eq. (3.2) simplifies to

i.e.

$$
T_{i 4}+\mu g_{i 4}=0,
$$

$$
\left\{\begin{array}{l}
T_{4}^{\alpha}=0, \\
T_{44}=-\mu g_{44},
\end{array}\right.
$$

${ }^{8}$ The only notable exception to Eq. (3.2) arises in the case of a material continuum interacting with an electromagnetic field. To this purpose, see Subsection 3.3. 
while Eq. (3.3) is identically satisfied. Conversely, in view of the assumed non-degeneracy of the eigenvalue $\mu$, it may be easily seen that Eq. (3.10a) is mathematically equivalent to Eq. (3.1) through the eigenvalue equation (3.2). We may thus regard Eq. (3.10a) itself as an alternative characterization of the co-moving frame of reference $[\Gamma]$.

Also, by Eqs. (3.5), (3.8) we obtain

$$
S_{i 4}=0, \quad S_{\alpha \beta}=\gamma_{\alpha \mu} \gamma_{\beta \nu} S^{\mu \nu},
$$

which shows that the stress tensor $S_{i j}$ is a space tensor in $[\Gamma]$.

Finally, by Eqs. $\left(3.6^{\prime}\right),\left(3.7^{\prime}\right),(3.8)$ we obtain

$$
\begin{gathered}
\lambda_{\xi}=\lambda_{\xi}\left(\mu, g_{i j}\right), \\
S_{i j}=S_{i j}\left(\mu, g_{i j}\right),
\end{gathered}
$$

while Eq. (3.4) becomes

$$
T_{i j}=\mu \gamma_{i} \gamma_{j}-S_{i j}\left(\mu, g_{i j}\right) .
$$

Eqs. (3.8)-(3.14) describe the properties of the given continuum in the co-moving frame of reference. To discuss the problem of motion we have still at our disposal Einstein's gravitational equations

$$
G_{i j}=-\kappa T_{i j},
$$

and a suitable set of initial and boundary data specified on the hypersurfaces $B$ and $S$ defined in Subsection 3.1.

Eqs. (3.10a, b), (3.15) imply

$$
\left\{\begin{array}{l}
G_{4}^{\alpha}=0, \\
G_{44}=\kappa \mu g_{44} .
\end{array}\right.
$$

Eq. (3.16b) relates the density $\mu$ of the continuum to the gravitational fields $g_{i j}$.

Moreover, according to the discussion following Eqs. (3.10a, b), we see that Eq. (3.16a) are mathematically equivalent to Eq. (3.1) through the field equation (3.15) and the eigenvalue equation (3.2). We may thus take Eq. (3.16a) as the relevant co-ordinate conditions that identify the co-moving frame of reference $[\Gamma]$. If we compare these facts with Definition 3.1, we see that Eqs. $(3.16 \mathrm{a}, \mathrm{b})$ satisfy the condition (i) stated there. However, it is easily verified that they do not satisfy condition (ii), as both equations reduce to identities in the limit $\kappa \rightarrow 0\left(\Rightarrow G_{i j} \rightarrow 0\right)$.

We can therefore state

Theorem 3.2. In the co-moving scheme, the determination of the equations of motion for a material continuum satisfying the eigenvalue equation (3.2) is reduced to the following problem: to find a system of $3+1$ equations, mathematically equivalent to Eqs. (3.16a, b), and having the property of being non trivial in the limit $\kappa \rightarrow 0$. 


\subsection{The Einstein-Maxwell System}

We now come to the only notable case in which Eq. (3.2) is violated, namely the case of a material continuum interacting with an electromagnetic field. To avoid unnecessary complications, we restrict our analysis to a charged incoherent dust, i.e. to an energy-momentum tensor of the simple form

$$
T_{i j}=\mu V_{i} V_{j}+\tau_{i j}
$$

Here $\tau_{i j}$ denotes the electromagnetic energy-momentum tensor, which is defined in terms of the electromagnetic tensor $F_{i j}$ by

$$
\tau_{i j}=F_{i p} F_{j}^{p}-\frac{1}{4} g_{i j}\left(F_{p q} F^{p q}\right) .
$$

The tensor $F^{i j}$ is antisymmetric, and satisfies the Maxwell equations

with

$$
\left\{\begin{array}{l}
F^{i j}{ }_{/ j}=J^{i} \\
* F^{i j} / / j=0
\end{array}\right.
$$

$$
J^{i}=\sigma V^{i}
$$

$\sigma$ denoting the charge density of the dust, and ${ }^{*} F^{i j}=\frac{1}{2} \eta^{i j k m} F_{k m}$ being the dual of the tensor $F^{i j}$.

As usual, we analyse the system $(3.17) \div(3.20)$ in the world-tube $\Omega$ introduced in Subsection 3.1.

To this purpose, we consider the complex tensor field

$$
M^{i j}=F^{i j}+i * F^{i j}
$$

In terms of this field, Eqs. $(3.19 \mathrm{a}, \mathrm{b})$ may be synthesized in the single system

$$
M^{i j}{ }_{/ / j}=J^{i} \text {. }
$$

By the antisymmetry of $M^{i j}$, this gives

$$
\left(\sqrt{|g|} M^{i j}\right)_{, j}=\sqrt{|g|} J^{i} .
$$

For $i=4$, Eq. (3.22') imply

$$
\left(\sqrt{|g|} M^{4 \alpha}\right)_{, \alpha}=\sqrt{|g|} J^{4} .
$$

Eq. (3.23) does not involve any time derivative; therefore, on the initial hypersurface $B$, it constitutes a consistency condition for the Cauchy data.

Theorem 3.3. Let the consistency condition (3.23) be satisfied on $B$. Then the system (3.22) is mathematically equivalent to

$$
\left\{\begin{array}{l}
\left(\sqrt{|g|} M^{\alpha j}\right)_{, j}=\sqrt{|g|} J^{\alpha}, \\
J^{i}{ }_{/ / i}=0
\end{array}\right.
$$

in the whole domain $\Omega$. 
Proof. Obviously Eq. (3.22) imply Eqs. (3.24a, b), as, in view of the antisymmetry of $M^{i j}$, we have

$$
M^{i j}{ }_{/ j i} \equiv 0 .
$$

Conversely, assume Eqs. (3.24a, b) and the consistency conditions (3.23) on $B$. Then, by Eqs. $(3.23) \div(3.25)$, the vector field $W^{i \text { def }}=M^{i j}{ }_{/ j}-J^{i}$ satisfies

$$
\begin{gathered}
\left\{\begin{array}{l}
W^{\alpha}=0 \\
W^{i}{ }_{/ / i}=0
\end{array} \quad \text { in } \Omega,\right. \\
W^{4}=0 \quad \text { on } B .
\end{gathered}
$$

It may be easily verified that Eqs. (3.26) $\div(3.28)$ admit the unique solution $W^{i}=0$, i.e. $M^{i j}{ }_{1 / j}=J^{i}$ in the whole domain $\Omega$. This completes the proof of Theorem 3.3.

We may thus regard Eqs. (3.20), (3.24a, b) and the consistency condition (3.23) as our basic system of electromagnetic field equations in $\Omega$.

In the co-moving scheme, this system is greatly simplified by the condition $V^{i}=\gamma^{i}$. In fact, in this case, Eq. (3.20) implies

$$
J^{\alpha}=0 ; \quad J^{4}=\sigma \gamma^{4}=\frac{\sigma}{\sqrt{-g_{44}}}
$$

so that Eqs. $(3.24 \mathrm{a}, \mathrm{b})$ reduce to

$$
\begin{gathered}
\left(\sqrt{|g|} M^{\alpha j}\right)_{, j}=0, \\
J_{, 4}^{4}+J^{4} \log (\sqrt{|g|})_{, 4}=0 .
\end{gathered}
$$

If we denote by $\sigma_{0}$ and $\gamma_{0}$ the initial values of $\sigma$ and $\gamma\left(=\operatorname{det} \gamma_{\alpha \beta}\right)$ on the hypersurface $B$, and make use of Eq. (3.29) and of the identity $\gamma^{4} \sqrt{|g|}=\sqrt{\gamma}$ we see that Eq. (3.31) leads immediately to

$$
\sigma=\frac{\sigma_{0} \sqrt{\gamma_{0}}}{\sqrt{\gamma}} .
$$

Eq. (3.31) expresses the conservation law for the electric charge in the co-moving scheme; in particular, it shows that the charge density $\sigma$ in $\Omega$ is uniquely determined in terms of the gravitational fields $g_{i j}$ and of the initial data.

We are still left with the differential system (3.30). In order to analyse its mathematical structure, it is convenient to introduce the complex space vector

$$
\tilde{M}_{\alpha}=\tilde{E}_{\alpha}+i \tilde{H}_{\alpha}=\frac{F_{4 \alpha}}{\sqrt{-g_{44}}}+\frac{1}{2} i \tilde{\eta}_{\alpha \lambda \mu} F^{\hat{\lambda} \mu}
$$


whose real and imaginary part are respectively the electric and the magnetic field associated to the electromagnetic tensor $F^{i j}$ in the comoving frame of reference $[\Gamma]^{9}$.

Theorem 3.4. The components $M^{i j}$ are uniquely expressed in terms of $\tilde{M}_{\alpha}$ in the linear form

with

$$
M^{i j}=C^{i j \alpha} \tilde{M}_{\alpha}
$$

$$
C^{i j \alpha}=\left(\gamma^{i} g^{\alpha j}-\gamma^{j} g^{\alpha i}\right)-i\left[\delta_{\mu}^{i} \delta_{v}^{j}+\left(\gamma^{i} \delta_{v}^{j}-\gamma^{j} \delta_{v}^{i}\right) \gamma_{\mu}\right] \tilde{\eta}^{\mu \nu \alpha} .
$$

We omit the proof of Theorem 3.4, as it is easily obtained by direct computation.

It is now convenient to introduce four complex matrices $C^{j}$ of order 3 , whose entries are given by

$$
\left(\hat{C}^{j}\right)^{\alpha \beta} \stackrel{\text { def }}{=}-\sqrt{|g|} C^{\alpha j \beta} .
$$

We have then, by Eqs. (3.35), (3.36)

$$
\begin{gathered}
\left(\hat{C}^{\lambda}\right)^{\alpha \beta}=-i \sqrt{|g|} \tilde{\eta}^{\lambda \alpha \beta}, \\
\left(\hat{C}^{4}\right)^{\alpha \beta}=\gamma^{4} \sqrt{|g|}\left(g^{\alpha \beta}-i \gamma_{\mu} \tilde{\eta}^{\mu \alpha \beta}\right)=\sqrt{\gamma}\left(g^{\alpha \beta}-i \gamma_{\mu} \tilde{\eta}^{\mu \alpha \beta}\right) .
\end{gathered}
$$

Eqs. (3.37), (3.38) show that all the matrices $\hat{C}^{j}$ are hermitian, and that $\hat{C}^{4}$ is positive definite (check).

Moreover, Eqs. (3.34), (3.36) imply

$$
-\sqrt{|g|} M^{\alpha j}=\left(\hat{C}^{j}\right)^{\alpha \beta} \tilde{M}_{\beta} .
$$

Coming back to the system (3.30), we see by Eq. (3.39) that it may be written in matrix form as

$$
\left(\hat{C}^{j} \tilde{M}\right)_{, j}=\hat{C}^{j} \tilde{M}_{, j}+\hat{C}^{j},{ }_{j} \tilde{M}=0 .
$$

In view of the stated properties of the matrices $\hat{C}^{j}$, the system (3.40) is linear, hyperbolic and hermitian.

Systems of this kind obey a general uniqueness theorem, analogous to the one valid for symmetric hyperbolic systems in the real field $[1,14,15]$.

Here we only state the final result, which arises from a slight modification of the method shown in Ref. [14] for the symmetric hyperbolic case: the system (3.40) admits a unique solution $\tilde{M} \in C_{1}(\Omega)$ consistent with the initial and boundary data if and only if

(i) the value of $\tilde{M}$ is given on the initial hypersurface $B$,

(ii) the boundary conditions are sufficient to ensure that, if $\tilde{M}^{(1)}$ and $\tilde{M}^{(2)}$ are any two allowable values for $\tilde{M}$ on the hypersurface $S$, the

${ }^{9}$ We let the reader verify that the fields $\tilde{E}_{\alpha}$ and $\tilde{H}_{\alpha}$ defined by Eq. (3.33) are indeed space vectors in $[\Gamma]$. 
Poynting vector $P^{\lambda}=\tilde{\eta}^{\lambda \alpha \beta}\left(\tilde{E}_{\alpha}^{(2)}-\tilde{E}_{\alpha}^{(1)}\right)\left(\tilde{H}_{\beta}^{(2)}-\tilde{H}_{\beta}^{(1)}\right)$ associated to the difference $\tilde{M}^{(2)}-\tilde{M}^{(1)}$ satisfies $P^{\lambda} n_{\lambda} \geqq 0$ at every point of $S\left(n_{\lambda}\right.$ being the outgoing normal to $S$ ).

The previous results show that, whenever the initial and boundary data are suitably chosen (i.e. whenever the problem in study is well posed) the electromagnetic field $F^{i j}$ and the charge density $\sigma$ in the co-moving frame of reference are uniquely determined in terms of the gravitational fields $g_{i j}$ (and of course, of the initial and boundary data themselves) by Eqs. (3.40) and (3.32) respectively.

In the co-moving scheme we are thus entitled to write

$$
\begin{gathered}
\sigma=\sigma\left(g_{i j}\right), \\
F_{i j}=F_{i j}\left(g_{i j}\right)
\end{gathered}
$$

and also, by Eqs. (3.17), (3.18)

$$
T_{i j}=\mu \gamma_{i} \gamma_{j}+\tau_{i j}\left(g_{p q}\right) .
$$

If we now want to proceed to the study of the problem of motion, we have still at our disposal Einstein's gravitational equations (3.15).

An argument similar to the one already used in Subsection 2.2 then shows that Eqs. (3.15), (3.43) imply

$$
\left\{\begin{array}{l}
G_{4}^{\alpha}+\kappa \tau_{4}^{\alpha}\left(g_{i j}\right)=0, \\
G_{44}+\kappa \tau_{44}\left(g_{i j}\right)=\kappa \mu g_{44},
\end{array}\right.
$$

and that Eq. (3.44a) are mathematically equivalent to the co-ordinate conditions that identify the co-moving frame of reference $[\Gamma]$.

Noticing that the left-hand sides of both Eqs. $(3.44 \mathrm{a}, \mathrm{b})$ depend only on $g_{i j}$, and that both equations reduce to identities in the limit $\kappa \rightarrow 0$ $\left(\Rightarrow G_{i j} \rightarrow 0\right)$, exactly as in Subsection 2.2 we conclude.

Theorem 3.5. In the co-moving scheme, the determination of the equations of motion for a charged dust is reduced to the following problem: to find a system of $3+1$ equations, mathematically equivalent to Eqs. $(3.44 \mathrm{a}, \mathrm{b})$, and having the property of being non-trivial in the limit $\kappa \rightarrow 0$.

\section{$\S$ 4. The Equations of Motion in the Co-moving Scheme}

We now come to the only problem which is still left open after the analysis of Section 3, namely the determination of the equations of motion for the given continuum in the co-moving scheme. 
To this purpose, we consider again the world-tube $\Omega$ and the hypersurfaces $B$ and $S$ introduced in Subsection 3.1. We have then

Theorem 4.1. Let $W^{i j} \in C_{1}(\Omega)$ be a symmetric tensor field satisfying

$$
\begin{array}{rlll}
W_{i}^{4}=0 & \text { on } & B, \\
W^{i j} f_{, j}=0 & \text { on } & S .
\end{array}
$$

Then, in the domain $\Omega$, the following statements are mathematically equivalent:
( $\alpha) \quad W_{i j}=0$

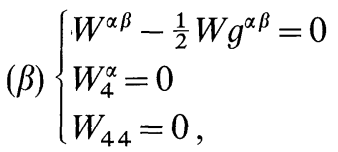
$(\gamma)\left\{\begin{array}{l}W^{\alpha \beta}-\frac{1}{2} W g^{\alpha \beta}=0 \\ W_{4}^{\alpha}=0 \\ W_{4 / / j}^{j}=0,\end{array}\right.$
$(\delta)\left\{\begin{array}{l}W^{\alpha \beta}-\frac{1}{2} W g^{\alpha \beta}=0 \\ W_{44}=0 \\ W^{\alpha j}{ }_{/ / j}=0,\end{array}\right.$

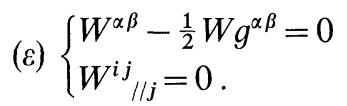

The proof of Theorem 4.1 follows directly from the results contained in Lemma 2.1 and Lemma 2.2 of Ref. [1], applied to the special case $V^{i}=\gamma^{i}$. We leave the details of the proof as an exercise to the reader.

If we now make the identification

$$
W^{i j}=G^{i j}+\kappa T^{i j},
$$

the conditions (4.1), (4.2) are necessarily fulfilled, and are in fact to be regarded as consistency conditions for the initial and boundary data $[1,10]$. We can thus replace Eqs. $(\alpha)$ - which, in view of Eq. (4.3), are now Einstein's gravitational equations - by any of the systems $(\beta),(\gamma)$, $(\delta),(\varepsilon)$

Let us consider the system $(\beta)$ first. In the co-moving scheme, comparison with Eqs. $(3.10 \mathrm{a}, \mathrm{b})$ and $(3.44 \mathrm{a}, \mathrm{b})$ yelds

$$
\left\{\begin{array}{l}
R^{\alpha \beta}=-\kappa\left(T^{\alpha \beta}-\frac{1}{2} T g^{\alpha \beta}\right), \\
G_{4}^{\alpha}=0, \\
G_{44}=\kappa \mu g_{44}
\end{array}\right.
$$


in the purely gravitational case, and

$$
\left\{\begin{array}{l}
R^{\alpha \beta}=-\kappa\left(T^{\alpha \beta}-\frac{1}{2} T g^{\alpha \beta}\right) \\
G_{4}^{\alpha}+\kappa \tau_{4}^{\alpha}=0 \\
G_{44}+\kappa \tau_{44}=\kappa \mu g_{44}
\end{array}\right.
$$

for a charged dust.

Comparison of Eqs. $(4.4 \mathrm{a}, \mathrm{b}, \mathrm{c}),(4.5 \mathrm{a}, \mathrm{b}, \mathrm{c})$ with Theorems 3.2 and 3.5 implies.

Theorem 4.2. Let the symmetric tensor field $W^{i j}$ be defined by Eq. (4.3). Then, in the co-moving scheme, the system $(\beta)$ of Theorem 4.1 consists of the equation

$$
R^{\alpha \beta}=-\kappa\left(T^{\alpha \beta}-\frac{1}{2} T g^{\alpha \beta}\right)
$$

and of a set of $3+1$ equations that are mathematically equivalent to the equations of motion for the given continuum, whether or not on electromagnetic field is present.

Corollary 4.1. In the co-moving scheme, the equations of motion for the given continuum are the divergence equations

$$
T^{i j}{ }_{/ / j}=0 \text {. }
$$

More precisely, the equation

$$
T_{4 / / j}^{j}=0
$$

relates the density $\mu$ of the continuum to the gravitational fields $g_{i j}$, while the equations

$$
T^{\alpha j} / / j=0
$$

constitute a set of co-ordinate conditions for the co-moving frame of reference $[\Gamma]$.

Proof. The fact that Eq. (4.7) are mathematically equivalent to the equations of motion is an immediate consequence of Theorems 4.1 and 4.2 , and of the Bianchi identities $G^{i j} / j=0$. Moreover, it is easily seen that Eq. (4.7) remain nontrivial in the limit $\kappa \rightarrow 0$. Therefore, by Definition 3.1, Eq. (4.7) are indeed the equations of motion for the given continuum.

The second assertion of Corollary 4.1 depends on the fact that, always in view of Theorem 4.1, Eq. (4.8) is mathematically equivalent to Eq. (4.4c) (or to Eq. (4.5c)), while Eq. (4.9) are mathematically equivalent to Eq. (4.4 b) (or to Eq. (4.5 b)).

However, this fact may also be seen directly. In fact, according to the results shown in Section 3 (Eqs. (3.14), (3.43)), in the co-moving scheme $23 *$ 
we are always entitled to write

$$
T_{i j}=T_{i j}\left(\mu, g_{i j}\right) .
$$

We may thus use Eqs. (4.8), (4.10) and the initial and boundary data to express $\mu$-and therefore also $T_{i j}$-as functions of $g_{i j}$.

Once this has been done, Eq. (4.9) involve the metric tensor only, so that they are indeed a set of co-ordinate conditions. The previous arguments show that Eq. (4.9) are in fact the co-ordinate conditions that identify the co-moving frame of reference $[\Gamma]$.

The proof of Corollary 4.1 is thus complete.

We conclude this Section by discussing how one could - at least in principle - determine the gravitational fields in the co-moving scheme, and thus obtain an effective solution of the problem of motion.

We have already noticed that, by means of Eqs. (4.8), (4.10), we may express the energy-momentum tensor $T_{i j}$ in the form $T_{i j}=T_{i j}\left(g_{i j}\right)$.

Once this has been done, we have still at our disposal the three co-ordinate conditions (4.9), and the remaining set of gravitational equations (4.6), i.e. a system of 9 independent equations involving the ten unknown components $g_{i j}$ only.

The indeterminacy of this situation is only apparent, as the whole formalism related to the co-moving scheme is obviously invariant under the group $\mathscr{I}$ of internal transformations, so that we cannot expect a unique determination of $g_{i j}$ in the co-moving frame of reference.

In practice, however, we may always remove this indeterminacy by adding one extra co-ordinate condition, able to select a particular atlas $\mathscr{A}$ in $[\Gamma]$.

As a final remark, we notice that we may now answer the question raised in Section 3.1 (footnote 6) on the legitimacy of the assumption $\Gamma \in\left(C_{2}, \hat{C}_{4}\right)$. In fact, this assumption is valid if and only if the co-moving frame of reference $[\Gamma]$ is not empty, i.e. if $[\Gamma]$ contains at least one admissible atlas $\mathscr{A}$. This requires that the class $\left\{g_{i j}\right\}$ of solutions obtained by means of the co-moving scheme contains at least a field $g_{i j} \in\left(C_{1}, \tilde{C}_{3}\right)$.

The previous arguments show that this condition is surely satisfied provided that the equations describing the internal structure of the continuum and the initial and boundary data are sufficiently smooth (at least $\left(C_{1}, \tilde{C}_{3}\right)$ ).

Part of this work has been performed while the Author was a scholar at the Dublin Institute for Advanced Studies, School of Theoretical Physics. The Author wishes to express his sincere gratitude to Prof. J. L. Synge and to Prof. J. R. Mc Connell for their warm hospitality, and to Prof. P. C. Vaidya for many helpful discussions. 


\title{
Bibliography
}

1. Massa,E.: Commun. math. Phys. 20, 279 (1971).

2. Landau,L.D., Lifshitz,E. M.: Fluid Mechanics. London: Pergamon Press 1959.

3. Israel, W.: Proc. Roy. Soc. 259, 129 (1960).

4. Courant, R., Friedrichs, K. O.: Supersonic Flow and Shock Waves-New York: Interscience 1948.

5. Møller,C.: The Theory of Relativity. Oxford: Clarendon Press 1952.

6. Cattaneo, C.: Nuovo Cimento 10, 318 (1958).

7. - Nuovo Cimento 11, 733 (1959).

8. - Nuovo Cimento 13, 237 (1959).

9. Massa, E.: Nuovo Cimento B 42, 178 (1966).

10. Lichnerowicz, A.: Thèories Relativistes de la Gravitation et de l'Èlectromagnetisme. Paris: Masson 1955.

11. Sternberg, S.: Lectures on Differential Geometry. Englewood/Cliffs, N.J.: Prentice Hall 1964.

12. Synge, J. L.: Relativity, the General Theory. Amsterdam: North Holland 1960.

13. Fock, V.A.: The Theory of Space, Time and Gravitation. Moscow: Pergamon Press 1964.

14. Courant, R., Hilbert, D.: Methods of Mathematical Physics, Vol. II. New York: Interscience 1962.

15. Massa, E.: Commun. math. Phys. 12, 246 (1969).

\author{
Enrico Massa \\ Istituto Matematico \\ Via L. B. Alberti, No. 4 \\ Università di Genova \\ I-16132 Genova, Italy
}


